\title{
ECONOMÍA VERDE: UN BREVE ANÁLISIS DE LOS BENEFICIOS DE SU APLICACIÓN
}

\section{GREEN ECONOMY: A BRIEF ANALYSIS OF THE BENEFITS OF ITS APPLICATION}

\author{
Marcos Gregorio Sánchez Calderón \\ mgsanchez7@espe.edu.ec \\ Universidad de las Fuerzas Armadas - ESPE, Salinas - Ecuador
}

\section{RESUMEN}

Una economía verde es aquella que tiene bajas emisiones de carbono, utiliza los recursos naturales de forma eficiente y es socialmente incluyente; la sociedad en su conjunto debe asumir que el proceso de transición ya empezó, pero se requiere un mayor impulso de los sectores público y privado. Los primeros pasos para ampliar y fortalecer el enverdecimiento de una economía es dar valor al capital natural, modificar y fortalecer el marco regulatorio para las inversiones, utilizar de forma eficiente los recursos naturales y crear puestos de trabajo verdes. Como consecuencia de una utilización eficiente del capital humano y natural existente en el planeta se optimiza el desarrollo económico, se mitiga las desigualdades sociales y se reorienta el nuevo rumbo del mundo. Estos aspectos son tratados a continuación para sustentar los beneficios de su aplicación.

Palabras clave: capital natural, sostenibilidad, enverdecimiento, economía verde.

\section{ABSTRACT}

A green economy is one that has low carbon emissions, used natural resources efficiently and is socially inclusive; society as a whole must assume that the transition process has already started, but a boost higher from the public and private sectors is required. The first steps to expand and strengthen the greening of an economy is to give value to the natural capital, modify and strengthen the regulatory framework for the investments, efficiently use natural resources and create green jobs. As a result of efficient utilization of human and natural capital on the planet is optimized economic development, social inequality is mitigated and the new direction of the world is reoriented. These aspects are discussed below to support the benefits of its application.

Keywords: natural capital, sustainability, greening, green economy.

Recibido: diciembre de 2015 Aprobado: mayo de 2016

\section{Introducción}

La histórica "Cumbre de la Tierra" tuvo lugar en Río de Janeiro, Brasil, el año 1992, veinte años después, se realizó la Cumbre de Desarrollo Sostenible de Naciones Unidas, denominada "Río+20", donde se definieron los lineamientos para la transición del planeta hacia una "economía verde" basada en un modelo de desarrollo económico con erradicación de la pobreza y protección ambiental. A partir de este año (2012) se habla con mayor frecuencia sobre la economía verde, convirtiéndose en un nuevo paradigma que surge tras el fracaso del modelo económico dominante; ahora, el reto es obtener riqueza con un impacto ambiental que minimice los riesgos, las carestías ecológicas y las disparidades sociales.

En este contexto, Rivas (1997, p.75) habla de implementar "un nuevo sistema de relaciones internacionales y de un desarrollo sostenible ecológicamente, y ética y socialmente admisible, para lo cual, es necesario mejorar las políticas públicas, incluyendo medidas de fijación de precios y reguladoras, para cambiar los incentivos de mercado perversos que hacen que el capital se asigne de manera inadecuada y que se ignoren las externalidades sociales y ambientales."

En este estudio se analizan tres temas que pueden considerarse como los más influyentes para lograr la transición hacia una economía verde. El primer tema, se refiere a que una economía verde concede valor al capital natural, conformado por los ecosistemas y la diversidad biológica del planeta, lo cual 
requiere una reorientación de las inversiones pública y privada, que permitan transformar sectores claves de la sociedad. El segundo tema, tiene que ver con la erradicación de la pobreza y la creación de nuevos empleos que están inevitablemente ligados al mantenimiento y conservación de los bienes ecológicos comunes, en sectores como: agricultura, silvicultura, agua dulce, pesca y energía, para que los beneficios por la utilización del capital natural se direccionen a los sectores más afectados. El tercer tema, promueve el uso eficiente de los recursos naturales, y fomenta una vida urbana más sostenible, siendo necesario que los planificadores y gobernantes nacionales y regionales diseñen políticas claras y graduales que encaucen las actividades hacia esa dirección.

\section{¿Qué es la economía verde?}

En marzo de 2009 se publicó el informe "Global Green New Deal. Policy Brief" que sirvió como base para un nuevo informe del Programa de las Naciones Unidades para el medio Ambiente (PNUMA en adelante) publicado en 2011 con el título "Hacia una economía verde: guía para el desarrollo sostenible y la erradicación de la pobreza. Síntesis para los encargados de la formulación de políticas", en ese documento se "considera que una economía verde debe mejorar el bienestar del ser humano y la equidad social, a la vez que reduce significativamente los riesgos ambientales y las escaseces ecológicas." (Bermann, 2012, p.11)

EI PNUMA (2011) explica que una economía verde seria aquella que tiene bajas emisiones de carbono, utiliza los recursos de forma eficiente y es socialmente incluyente, que los ingresos y la creación de empleos deben derivarse de inversiones públicas y privadas destinadas a reducir las emisiones de carbono y la contaminación, que debe promover la eficiencia energética así como en el uso de los recursos, y que debe evitar la pérdida de diversidad biológica y de servicios de los ecosistemas.

Para emprender la transición a una economía verde es necesario cumplir una serie de circunstancias favorables, que pueden ser:

- Re-direccionamiento de la inversión pública para el reverdecimiento de sectores claves;

- Aplicación de impuestos e instrumentos basados en el mercado para modificar las preferencias de los consumidores y estimular la inversión verde y la innovación;

- Reducción de subsidios con efectos dañinos para el ambiente;
- Implementación de temas ambientales en las adquisiciones públicas;

- Aplicación de medidas que fomenten la creación de empleos; y,

- El establecimiento de normas y reglamentos que beneficien el cuidado ambiental y generen beneficios económicos a largo plazo.

Ejemplos de estas regulaciones con efecto mundial son la Cumbre Río+20 (Junio 2012), donde fueron aprobados dos importantes documentos para la biodiversidad mundial: la Agenda $21 \mathrm{y}$ el Convenio sobre la Diversidad Biológica; y, la Cumbre de París COP 21 (Diciembre 2015), en la que se llegó a un compromiso vinculante y definitivo para la reducción de emisiones contaminantes a la atmósfera a partir del año 2020 , cuyo propósito principal es limitar el aumento de la temperatura media mundial muy por debajo de $2^{\circ} \mathrm{C}$ con respecto a los niveles preindustriales, y de seguir esforzándose por limitar el aumento de la temperatura a $1,5^{\circ} \mathrm{C}$ (Figura 1$)$.
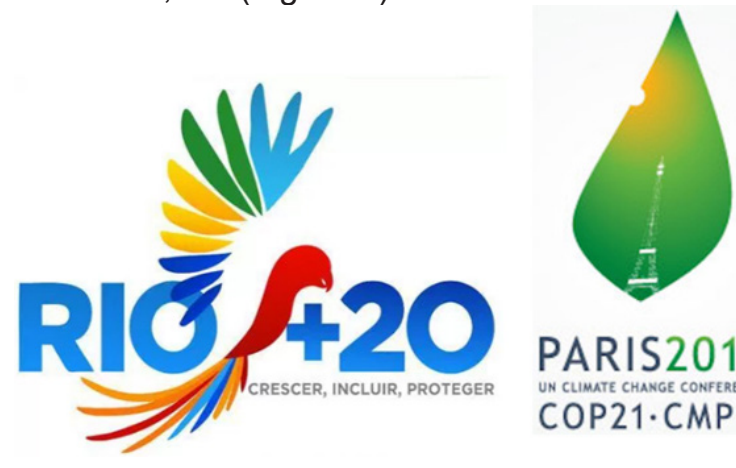

PARIS2015 UN CUMATE CHANGE CONFERENCE

Figura 1. Logotipos Cumbres Río+20 y París COP 21.

Considerar que la sostenibilidad ambiental tiene repercusiones negativas en el crecimiento económico[1] es un argumento falso, el enverdecimiento de las economías no impide crear riqueza ni generar empleos nuevos, y son muchos los sectores verdes que ofrecen interesantes oportunidades de inversión y de crecimiento en términos de riqueza y puestos de trabajo.

Otro mito, según comenta Michael (1996), es pensar que la economía verde es un lujo que solo pueden permitirse los países ricos o, lo que es peor, que se trata de una estrategia con la que el mundo desarrollado pretende impedir el desarrollo y perpetuar la pobreza de los países en desarrollo. Este argumento es falso, el hecho de que la gente pobre no pueda darse el lujo de comprar una buena calidad de vida no significa que no la quiera, un buen ejemplo es creer que la demanda que se registra en el mercado es la cabal expresión de los deseos de los consumidores; la demanda del 
mercado se basa en la demanda de los consumidores; pero los deseos, no siempre corresponden a los recursos que los consumidores tienen para satisfacerlos.

\section{La transición a una economía verde}

A continuación se analizan los principales hechos que se deben considerar para concretar la transición a una economía verde.

\section{La economía verde da valor al capital natural}

Para dar valor al medio ambiente es necesario comparar los costes y beneficios de las acciones adoptadas para su preservación, más aún, cuando los costes para minimizar los efectos de la degradación medioambiental y el cambio climático casi siempre son onerosos. Algunos de estos costes son, por ejemplo, el equipo (personas y maquinaria) que se requiere para el control de la contaminación excesiva, otros son los costos de no hacer cosas que podríamos haber hecho, como ocurre cuando no se explota un bosque para no sacar madera.

El capital natural que utiliza el sistema económico para la producción de bienes y servicios está compuesto por el petróleo, carbón, gas natural, madera, recursos hídricos y otros. Según Azqueta (2004) el capital natural se compone de una gran cantidad de elementos interrelacionados: activos naturales, activos ambientales, ecosistemas, etc., siendo su valor presente la parte principal.

Establecer valores económicos a los servicios de los ecosistemas, debería ser un compromiso de cada gobierno, para lo cual, es necesario que el entorno institucional[2] de un país se oriente hacia la sostenibilidad de la explotación. En seguida se comenta los aspectos más relevantes para su manejo y conservación:

- Las actuales tasas de deforestación y degradación de los bosques se deben a la demanda de productos madereros y a la presión causada por la agricultura y la ganadería; los países deberán ampliar los incentivos económicos globales y locales para reforestar, los beneficios que se obtendrían reduciendo a la mitad la deforestación mundial podrían ser, por si solos, tres veces superiores a los costos, según estudio realizado por The Eliasch Review (2008).

- Para enverdecer la agricultura, se requiere que los subsidios perjudiciales para el medio ambiente se vayan reduciendo y eliminando, esto implica un cambio en la matriz productiva agrícola industrial y de subsistencia, implementando prácticas de cultivo eficientes como el uso óptimo del agua, mejor preparación de los cultivos, utilización extensiva de nutrientes orgánicos y naturales para el suelo, y control de plagas. El enverdecimiento de este sector requiere "de activos de capital físico, inversiones financieras, investigación y desarrollo de capacidades en cinco áreas fundamentales: gestión de la fertilidad del suelo; utilización más eficiente y sostenible del agua; diversificación de cultivos y ganado; gestión biológica de la salud de plantas y animales; y, un nivel de mecanización agrícola apropiado." PNUMA (2011, p.15)

- El recurso agua requiere un tratamiento particular y especializado, ya que en su demanda intervienen los sectores: agrícola, industrial, y para consumo del hogar, que ejercen presión y tienen opuestos y complejos requerimientos en busca de alcanzar su satisfacción. "Para enverdecer el sector hídrico, se requiere invertir en los ecosistemas para mitigar su escasez y reformar las políticas hídricas, concentrándose en mejorar los acuerdos institucionales, los sistemas de concesión de derechos y de asignaciones, ampliar la aplicación de cobros por los servicios de los ecosistemas, reducir los subsidios a la inversión y mejorar el cobro por agua." Perelló (1996. p.33)

- El sector pesquero tiene que reducir su actual exceso de capacidad, mediante la reducción de subsidios y embarcaciones para recuperar las poblaciones de peces agotadas, lo que garantizará una fuente vital de ingresos a largo plazo, caso contrario el sector experimentará una contracción por el desplome de las poblaciones de peces.

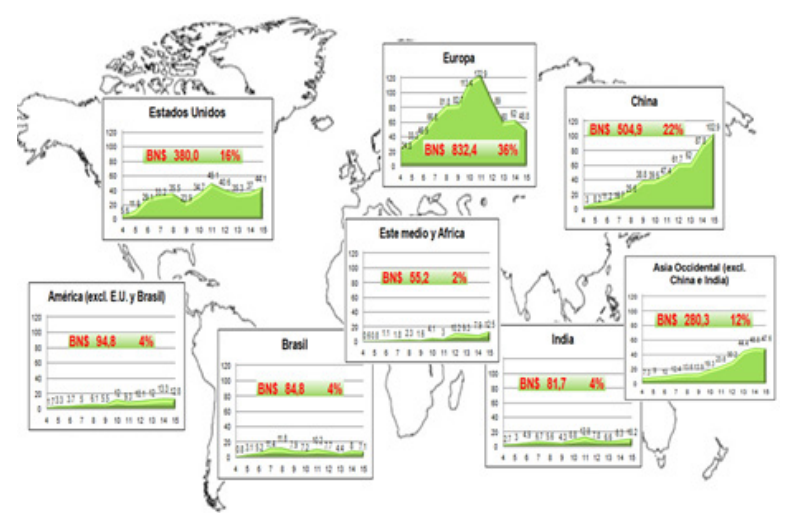

Figura 2. Inversión Mundial en Energías Renovables (Billones de dólares 2004-2015). Elaboración propia. Los datos están tomados del Global Trends in Renewable Energy Investment 2016.

Invertir en capital natural será la mejor forma de 
garantizar que el crecimiento y el empleo se mantengan, pues ello elevará las posibilidades de que los recursos naturales se conserven para el aprovechamiento de las futuras generaciones; un claro ejemplo es la inversión en energías renovables que se realizó en el planeta, periodo 2004-2015, por 2.314,10 billones de dólares (BN\$ en adelante); Europa destinó $832,40 \mathrm{BN} \$$, que representó la inversión más alta con cerca del $36 \%$ mundial, América (excluyendo Estados Unidos y Brasil) por su parte destinó 7,9 BN\$, que en términos relativos representaron alrededor del $4 \%$ mundial, esto la coloca, en término de bloques, en la quinta posición, muy por detrás de Estados Unidos, China, y Asia Occidental (Figura 2).

\section{La economía verde reduce la pobre- za y crea puestos de trabajo}

La economía verde es una de la actividades que está creciendo de forma lenta y sostenida, así como también es una de las mejores pagadas, por lo cual, tiene una proyección internacional muy interesante. La Organización Internacional del Trabajo (2012) considera que la transición hacia una economía más verde podría generar entre 15 y 60 millones de empleos adicionales en el mundo durante las próximas dos décadas y ayudar a decenas de millones de trabajadores a salir de la pobreza.

La economía verde busca potencializar las oportunidades de crecimiento económico que puede tener un país y al mismo tiempo mitigar la pobreza; entre las acciones más relevantes que una nación podría adoptar para proteger los activos naturales se recomienda lo siguiente:

- La agricultura sustentable mejora la calidad del medio ambiente y los recursos base de los cuales depende la agricultura; provee las fibras y alimentos necesarios para el ser humano; es económicamente viable y mejora la calidad de vida de los agricultores y la sociedad en su conjunto. Fortalecer la explotación agrícola en las pequeñas fincas, evitará la migración interna del campo a las ciudades y consecuentemente el costo que implica tener tierras improductivas, esta es considerada la medida más eficaz para enverdecer la agricultura en los países en desarrollo.

- El agua, siendo una necesidad básica para la vida, no es accesible para gran parte de la población en los países pobres, los costos de acceder a este servicio y remediar las enfermedades transmitidas por un tratamiento inadecuado del suministro de agua son muy altos, por lo que invertir en infraestructuras para obtener agua limpia y servicios de saneamiento eficientes, es una de las mejores maneras de acelerar la transición a una economía verde.

- El sistema energético actual es extremadamente desigual, invertir en energías renovables pueden ser una estrategia rentable para eliminar la pobreza y contribuir a mejorar el nivel de vida y la salud en las áreas de ingresos bajos, especialmente en las zonas más aisladas, ejemplos de su aplicación son la "biomasa limpia y la energía solar fotovoltaica no conectada a la red, que ofrecen costos de operación reducidos y flexibles y la posibilidad de utilizarlas a pequeña escala", según informe del PNUMA (2001, p.19).

- El turismo comprende temas como: comercialización, transporte, alojamiento y restauración, infraestructura y servicios, consecuentemente, no existe caso de desarrollo turístico que no cause ningún impacto sobre el medio ambiente, aspectos que deben considerarse para mitigar su efecto sobre los ecosistemas. (Rivas 1997)

El desarrollo del turismo, bien planificado, puede mejorar la economía local y reducir la pobreza, mediante un incremento en el número de contrataciones y adquisiciones; "se calcula que un puesto de trabajo en el sector turismo básico implica la creación de 1,5 puestos de trabajo adicionales o indirectos en la economía relacionada con el turismo", según Mesera (1999, p.14).

- Finalmente, los países que orientan su política hacia una economía verde están experimentando una mejora significativa en la creación de nuevos empleos, por citar algunos ejemplos: los estímulos para la creación de las Pymes[3], promover el uso de energías renovables, y la creciente mejora en la cultura de reciclaje.

Estudios de la OIT (2013, p.4) "demuestran que el empleo decente y medioambientalmente sostenible contribuye a la reducción de la pobreza, a la igualdad e inclusión social, al bienestar humano, al desarrollo económico y a la protección del medio ambiente." A manera de ejemplo, se explica el caso español sobre empleos verdes, estas actividades emplean a unas 530 mil personas en actividades principales y relacionadas, que en términos relativos representó el 2,2\% del empleo total de la economía española en el año 2009. Según la distribución sectorial que se muestra en la Tabla 1, el sector que más empleos generó es la recogida y tratamiento de residuos con $140 \mathrm{mil}$ empleos (26\% del total); le sigue el sector de las energías renovables, con 110 mil empleos (21\%), 
y el sector de tratamiento y depuración de aguas residuales, con 58 mil trabajadores (11\%).

Tabla 1.

Distribución sectorial de empleos verdes en España (2009)

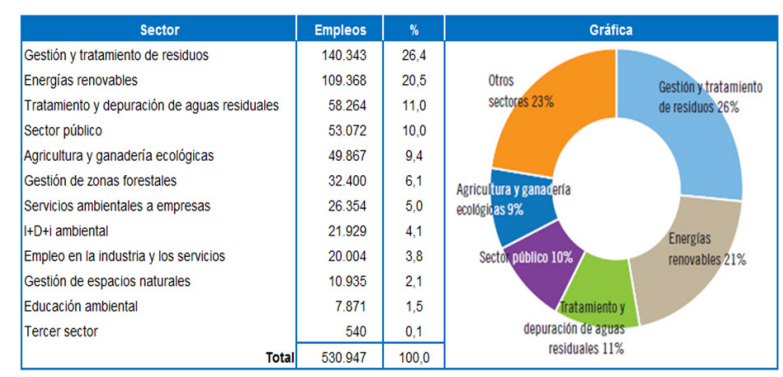

Fuente: Sustainlabour 2012. (Adaptación Tabla 4, p. 22)

\section{La economía verde motiva un uso eficiente de los recursos naturales y facilita una vida urbana más sos- tenible}

Conseguir un uso eficiente de los recursos naturales y optimizar los beneficios económicos que se derivan de él no es una tarea fácil. El crecimiento económico se ve comprometido por la escasez de recursos y está llevando al planeta a límites insostenibles.

A continuación, se analizan algunas alternativas que mitiguen el impacto ambiental, y promuevan la generación de riqueza originada por el uso eficiente de los recursos naturales:

- La manufactura es una etapa clave en el ciclo de vida de las materias primas, que comienza con la extracción de los recursos naturales y termina con su disposición final; en el proceso productivo se ocupa gran cantidad de agua, energía eléctrica y recursos primarios, consecuentemente, el crecimiento productivo viene acompañado de problemas de toxicidad por el uso de sustancias peligrosas y contaminación del aire. El sector debe aplicar economías de escala para aprovechar eficientemente los recursos, usando menos materias primas y recursos energéticos. Enverdecer el sector de la manufactura supone extender la duración de los productos, esto implica efectuar un rediseño para alargar su vida útil; otra alternativa es la re-manufactura que se basa en el reprocesamiento de productos y componentes usados, y la re-utilización que promueve el uso de subproductos y ofrece alternativas para sustituir determinados factores de la producción.

- El reciclaje y la recuperación de energía a par- tir de los residuos son actividades cada vez más rentables, una tendencia que se mantendrá a medida que los residuos se conviertan en recursos más valiosos, PNUMA (2011) señala que los flujos de residuos están asociados al incremento del nivel de vida, y es indudable que actualmente se recupera un pequeño porcentaje, siendo necesario la implementación de políticas regulatorias y de precios a nivel estatal para orientar a industrias y consumidores hacia un uso más eficiente de los recursos.

- Otro hecho que merece ser analizado es que el acelerado crecimiento de los núcleos urbanos que están ejerciendo presión sobre el suministro de agua dulce; los sistemas de alcantarillado y la salud pública a menudo son insuficientes e inadecuados, el equilibrio ambiental se afecta y se generan costos significativos para mitigar sus efectos. En este contexto, las ciudades deben reducir las emisiones y los residuos generados por los edificios, a continuación se comentan algunos temas que influyen en el propósito de aumentar la eficiencia energética y la productividad en los núcleos urbanos:

- Las eco-ciudades o ciudades verdes, se caracterizan normalmente por una mayor densidad de población, vivienda, empleo, comercio e instalaciones de recreación, están sujetas a ciertos límites para evitar la congestión, incluyen criterios de sostenibilidad que permitan alcanzar eficiencia en su planificación urbana.

- El sector de la construcción consume una cantidad significativa de materias primas, agua dulce y genera residuos sólidos; es el mayor emisor de gases de efecto invernadero, se estima que un tercio de la energía mundial se consume dentro de los edificios (PNUMA 2009). Este sector tiene un enorme potencial para reducir la contaminación del aire doméstico, al enverdecerlo se optimizaría el uso de estos recursos reflejándose a lo largo de su vida útil.

- Los hogares dependen de la política gubernamental para fortalecer sus economías domésticas, ya que se debe normar la construcción de nuevos edificios verdes y adaptar aquellos existentes, los que deberán cumplir determinadas normas de construcción sostenible para alcanzar eficiencia en el consumo energético y mejora de la salud ambiental.

- Tanto en el ámbito urbano y rural el transporte es responsable de más de la mitad del consumo mundial de combustibles fósiles líquidos y de casi un cuarto de las emisiones de dióxido 
de carbono ( $\mathrm{CO} 2)$ relacionadas con el consumo energético. Al respecto, Rivas (1997: p.242) señala que los muchos y graves efectos ambientales de carácter negativo que soporta el transporte están ligados a los impactos generados por sus infraestructuras y sus servicios. Las políticas que podrían enverdecer el transporte se orientan a los siguientes temas: "1) evitar o reducir los desplazamientos de personas, integrando la utilización del suelo y la planificación del transporte y fomentando la producción y el consumo locales; 2) adoptar medios más eficientes desde el punto de vista ambiental, tales como el transporte público y los medios no motorizados (para pasajeros), y el transporte ferroviario o por agua (para mercancías); y, 3) mejorar tecnológicamente vehículos y combustibles para reducir sus efectos negativos, a nivel social y ambiental, por kilómetro recorrido." (PNUMA, 2011, p.30)

Tabla 2.

Los 10 países que más contaminan en el Mundo y en América del Sur (2011)

\begin{tabular}{|c|c|c|c|c|c|}
\hline Mundo & \begin{tabular}{|c|} 
Toneladas \\
métricas de \\
(C2)
\end{tabular} & $\begin{array}{c}\text { Sodel total } \\
\text { mundial }\end{array}$ & América del Sur' & \begin{tabular}{|c|} 
Toneladas \\
métricas de \\
(C2)
\end{tabular} & $\begin{array}{l}\text { Sdel total } \\
\text { mundial }\end{array}$ \\
\hline China $^{2}$ & $9.019,8$ & 26,03 & Brasil & 439,4 & 1,27 \\
\hline Estados Unidos $^{2}$ & $5.305,6$ & 15,31 & Argentina & 190,0 & 0,55 \\
\hline Unión Europea (27) & $3.574,1$ & 10,32 & Venezuela & 188,8 & 0,54 \\
\hline India ${ }^{2}$ & $2.074,3$ & 5,99 & Chile & 79,4 & 0,23 \\
\hline Federación Rusa' & $1.808,1$ & 5,22 & Colombia & 72,4 & 0,21 \\
\hline Japón' & 1.187,7 & 3,43 & Perú & 53,1 & 0,15 \\
\hline Alemania' & 729,5 & 2,11 & Ecuador & 35,7 & 0,10 \\
\hline Corea (Sur) ${ }^{2}$ & 589,4 & 1,70 & Bolivia & 16,1 & 0,05 \\
\hline Irán ${ }^{2}$ & 586,6 & 1,69 & Urguay & 7,8 & 0,02 \\
\hline Indonesia ${ }^{2}$ & 564,0 & 1,63 & Paraguay & 5,3 & 0,02 \\
\hline
\end{tabular}

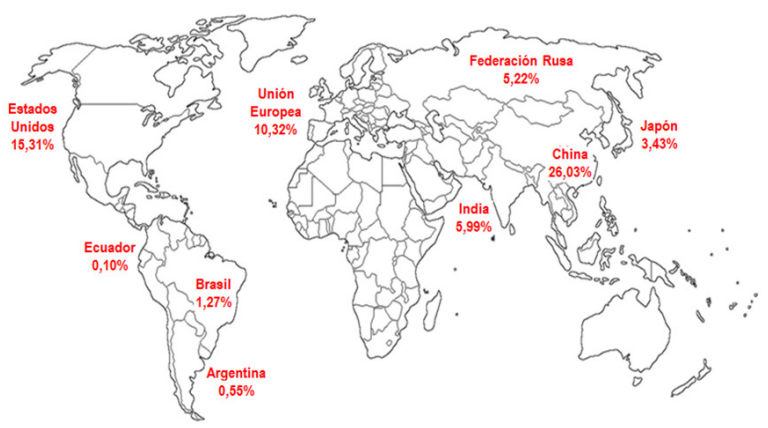

Fuente: Elaboración propia.

Los datos están tomados del Banco Mundial, Emisiones de CO2 (kt) 2011.

A manera de ejemplo, en la Tabla 2, se puede apreciar la lista de los 10 países que más contaminan a nivel mundial, China es responsable de la emisión del $26 \%$ de los gases de efecto invernadero, sin embargo, como país en vías de desarrollo, no está obligado a reducir las emisiones de $\mathrm{CO} 2$; Estados Unidos es el segundo más grande emisor de $\mathrm{CO} 2$ con un 15\% (cuando se firmó el Protocolo de Kioto en 1997 Washington se comprometió a reducir sus emisiones en un $6 \%$, poco después se retiró), los países miembros de la Unión Europea ratificaron el Protocolo de Kioto en mayo de 2002 y son responsables del $10 \%$ de las emisiones; India desplazó a Rusia del cuarto puesto con el $5,99 \%$ de emisiones de CO2. A nivel de América del Sur, el mayor emisor es Brasil, con $1,27 \%$, y Ecuador contribuye con el $0,10 \%$ del total mundial.

\section{Conclusiones}

Cada país debe adoptar las medidas necesarias para embarcarse en esta corriente de transición a una economía verde y sacar el máximo provecho de las ventajas de su aplicación; a continuación se formula las siguientes conclusiones:

Primera, se refiere a que los pilares del desarrollo sostenible, ambiente y sociedad, deben recibir similar trato que el económico; los motores de la sostenibilidad son los bosques y las fuentes de agua dulce, estos deben tener el mismo peso, si no mayor, cuando se realice la planificación económica y se adopten las políticas públicas de un país, es decir, una economía verde debe asignar valor al capital natural.

Segunda, la erradicación de la pobreza y la creación de nuevos empleos están inevitablemente ligados al mantenimiento y la conservación de los bienes ecológicos comunes, en sectores como: agricultura, silvicultura, agua dulce, pesca, energía y turismo. Los estados deberán garantizar que los beneficios generados por la utilización del capital natural se redistribuyan en los territorios donde se ejecutan proyectos de explotación de bienes ecológicos, lo que permitirá mejorar la calidad de vida de la población más desfavorecida.

Tercera, es promover el uso eficiente de los recursos naturales, sea mediante la reducción del impacto ambiental de un recurso natural, o reduciendo el volumen del recurso natural requerido para alcanzar bienestar, siendo necesario emitir políticas para lograr este cambio, que podrían ser: redistribuir regalías por la extracción de recursos no renovables, estimulación a la inversión en sectores claves, reducir o eliminar los subsidios que afectan el medio ambiente, compras públicas verdes, reducir o eliminar los subsidios perjudiciales para el medio ambiente, y un marco regulatorio adecuado.

En lo que respecta al Ecuador, se han dado avances significativos en la transición al enverdecimiento de su economía: tiene la primera Constitución del mundo que otorga derechos al medio ambiente, y en su planificación estratégica "Plan 
Nacional del Buen Vivir 2013-2017" considera temas como: "Garantizar los derechos de la naturaleza y promover la sostenibilidad ambiental territorial y global" y "Asegurar la soberanía y eficiencia de los sectores estratégicos para la transformación industrial y tecnológica", que actualmente están en fase de ejecución.

El propósito principal de realizar un análisis sobre los beneficios de la transición a una Economía Verde, es motivar tanto al sector académico científico, como a los sectores público y privado a profundizar en la investigación de este tema tan complejo, para que la sociedad en su conjunto se capacite y participe en el proceso, como paso previo para alcanzar un desarrollo sustentable e inclusivo.

\section{Notas}

[1] Existen muchos factores que explican el crecimiento económico de un país, entre los principales se pude señalar el capital físico, el capital humano, el conocimiento y tecnología, y la institucionalidad de un país; todos estos factores tienen que funcionar simultáneamente y de la manera más eficiente posible para que se dé el crecimiento. De ahí que la teoría del crecimiento económico es muy compleja.

[2] El entorno institucional de un país está conformado por las reglas de juego legales, las instituciones, la organización del gobierno y de sus funcionarios, inclusive el nivel de corrupción del mismo.

[3] Según el Servicio de Rentas Internas del Ecuador, se conoce como PYMES al conjunto de pequeñas y medianas empresas que de acuerdo a su volumen de ventas, capital social, cantidad de trabajadores, y su nivel de producción o activos presentan características propias de este tipo de entidades económicas. Por lo general, en el país las pequeñas y medianas empresas que se han formado realizan diferentes tipos de actividades económicas entre las que se subraya las siguientes:

- Comercio al por mayor y al por menor.

- Agricultura, silvicultura y pesca.

- Industrias manufactureras.

- Construcción.

- Transporte, almacenamiento, y comunicaciones.

- Bienes inmuebles y servicios prestados a las empresas.

- Servicios comunales, sociales y personales.

\section{Referencias Bibliográficas}

1. Azqueta, D., y Delacámara, G. El papel de las variables ambientales en la nueva geografía económica. Revista Investigaciones Regionales, 4, 145-175. (2004).

2. Banco Mundial. Indicador Emisiones de $\mathrm{CO} 2$ (kt). Recuperado de http://datos.bancomundial. org/indicador/EN.ATM.CO2E.KT/countries. (consultado el 7 de mayo del 2016). (2016).

3. Bermann, C. Economía verde: ¿una vía para otro mundo posible?. Revista Nueva Sociedad, 239, 11-23. (2012).

4. FS-UNEP Collaborating Centre. (2015). Global Trends in Renewable Energy Investment 2016. Recuperado de www.fs-unep-centre.org.

5. Mesera, O., Astier, M., y López, S. Sustentabilidad y manejo de Recursos Naturales. México: Mundiempresa. (1999).

6. Michael, J. La Economía Verde. Barcelona, España: Icaria. (1996).

7. Perelló, J. Economía Ambiental. Murcia. Alicante. Ediciones de la Universidad de Alicante. (1996).

8. OIT. La transición hacia una economía verde podría crear hasta 60 millones de empleos. (31 de mayo de 2012). Recuperado de http://www. ilo.org/global/about-the-ilo/newsroom/news/ WCMS_181808/lang--es/index.htm.

9. Los empleos verdes se vuelven realidad. Progreso y perspectivas 2013. Ginebra, Suiza. —. (2013).

10. Naciones Unidas. Convención Marco sobre el Cambio Climático. París, Francia. FCCC/ CP/2015/L.9. (2015).

11. - Asamblea General Sexagésimo sexto período de sesiones. Río de Janeiro, Brasil: 66/288. El futuro que queremos. (2012).

12. PNUMA. Sustainable Building Construction Initiative: (2009). Recuperado de http://www.unep. org/sbci/pdfs/UNEPSBCI-GlobalCompactBrochure-Final.pdf (consultado el 22 de diciembre del 2015).

13. - Hacia una economía verde: Guía para el desarrollo sostenible y la erradicación de la pobreza. Síntesis para los encargados de la formulación de políticas. Francia. (2011). Recuperado 
de www.unep.org/greeneconomy.

14. Rivas, D. Sustentabilidad: desarrollo económico, medio ambiente y biodiversidad. Madrid, España: Parteluz. (1997).

15. SENPLADES. Plan Nacional del Buen Vivir. Quito, Ecuador. (2013).

16. SELA. La visión de la economía verde en América Latina y el Caribe. Caracas, Venezuela: SP/Di No. 1-12, 1-52. (2012).

17. Sustainlabour. Empleos verdes para un desarrollo sostenible. El caso español. España: Paralelo Edición, S.A. (2012).

18. The Eliasch Review. Climate Change: Financing Global Forests. The Stationery Office Limited, Reino Unido. (2008).

19. UNEP \& New Energy Finance. (2010). Global Trends in Sustainable Energy Investment 2010. Recuperado de www.sefi-unep.org.

20. UNEP. Economía Verde en el contexto del desarrollo sostenible y erradicación de la pobreza: Una perspectiva desde América Latina y el Caribe. Quito, Ecuador. UNEP/LAC-IG.XVIII/3. (2012).

21. Yepes, A. Cambio Climático: estrategias de gestión con el tiempo en contra .... Universidad de los Llanos, Colombia, 16-1.

(2012). 